\title{
SPACE DISTRIBUTION OF CARBON STARS IN OUR GALAXY
}

\author{
K. NOGUCHI \\ National Astronomical Observatory of Japan \\ Mitaka, Tokyo 181, Japan \\ AND \\ Z. QIAN, J. SUN, G. WANG, J. WANG AND Y. RAO \\ Beijing Astronomical Observatory \\ Beijing 100080, P. R. China
}

\begin{abstract}
We are attempting to investigate the space distribution of carbon stars in our Galaxy by evaluating the distances of individual carbon stars. We estimate the distance by evaluating the total radiant energy of each star and assuming a value for the bolometric magnitude of carbon stars. Since the flux maxima of carbon stars are mostly in the near-infrared (NIR), NIR photometric data are most useful for evaluating the total energy. We have compiled NIR photometric data for 694 carbon stars at all galactic longitudes and have made new observations of 470 carbon stars in the galactic longitude region between $20^{\circ}$ and $160^{\circ}$. We discuss the space distribution of 1164 galactic carbon stars.
\end{abstract}

\section{Introduction}

The asymptotic giant branch (AGB) stars are classified into three main types on the basis of their atmospheric composition: carbon-rich, oxygenrich (O-rich) and S-type. It is interesting to compare the space distributions of these AGB stars in order to understand what kind of AGB stars evolve as carbon stars.

Jura (1990) reported that there is a measurable galactocentric gradient in the surface density (SD) projected onto the galactic plane of the Orich Miras, but that there is no decrease with galactocentric radius in the $\mathrm{SD}$ of carbon stars for at least $3 \mathrm{kpc}$ beyond the solar circle. The space distribution of carbon stars is different from that of O-rich AGB stars in 
the solar neighborhood. However, these investigations have been limited to the brighter carbon stars (Claussen et al. 1987; Jura \& Kleinmann 1989, 1990; Groenewegen et al. 1992).

A General Catalog of Cool Galactic Carbon Stars, 2d ed. (GCCS2), compiled by Stephenson (1989), includes 5987 carbon stars. The majority of these carbon stars are distributed near the galactic plane on the $l-b$ diagram. It is interesting to note that the stellar density of carbon stars is lower in the longitude range between about $-50^{\circ}$ and $+50^{\circ}$, i.e. in the direction towards the galactic center.

Guglielmo et al. (1993) reported near-infrared (NIR) photometric data for 1332 IRAS sources. Combining the measured $K-L$ color with the IRAS [12-25] color, they classified these sources into IRCS (Infrared Carbon Stars) and O-rich stars. On the basis of their data, they reported that the SD of IRCS is constant in the galactocentric distance range 5-11 kpc but might present a cut-off at about $4 \mathrm{kpc}$ from the Sun in the galactic center direction. However, further observations are required to clarify the global space distribution of carbon stars in our Galaxy, because the observations have been carried out only in limited regions of the Galaxy and their stellar classification is based only on infrared photometric data.

We have attempted a more extensive investigation of the space distribution of the carbon stars in GCCS2, which mostly are classified on the basis of optical spectra. How to estimate distances for individual stars is an important point in investigating the SD distribution. In this work, distances are determined by evaluating the total radiant energies of stars under an assumption of a certain bolometric luminosity for carbon stars. The space distribution of 694 relatively bright carbon stars is discussed in $\S 3$. The distribution of carbon stars, including 470 newly observed stars, is discussed in $\S 4$.

\section{Determination of Distances to Carbon Stars}

Distances to carbon stars are determined by comparing the observed radiant energy with the assumed intrinsic luminosity. All the carbon stars are assumed to be radiating with $10^{4} L_{\odot}$. Observed total radiant energies from individual stars are evaluated by integrating the spectral energy distributions (SEDs). In order to get SEDs of stars and to evaluate total radiant energies accurately, NIR photometric data are very important for carbon stars because the SEDs of the majority of carbon stars show their flux maxima in the NIR. Therefore, we estimated the distance only for stars with NIR photometric data. NIR photometric data and IRAS photometric data are used to get SEDs on $\lambda \cdot F_{\lambda}$ vs. $\lambda$ diagrams. Total radiant energies are evaluated by integrating the model spectra which are fitted to the 
observed SEDs.

Individual SEDs of carbon stars are fitted by either of two types of model spectra of carbon stars. Since the SEDs of relatively blue carbon stars are usually well fitted by blackbody spectra, 'blue' carbon stars are approximated by blackbody spectra. But red carbon stars, in most cases, have SEDs that are flatter than blackbody spectra. Therefore we made a model spectrum which fits better to the SEDs of red carbon stars on the average. In order to classify stars into blue or red classes, the $K-L$ color (or the wavelength where $\lambda \cdot F_{\lambda}$ shows its maximum when there is no $K-L$ data) is used.

\section{Distribution of Relatively Bright Carbon Stars}

We have compiled NIR $(J, H, K, L)$ photometric data for carbon stars in the GCCS2 from the observations reported by Catchpole et al. (1979), Epchtein et al. (1987, 1990), Guglielmo et al. (1993), and Noguchi et al. (1981, 1991). We added our unpublished NIR photometric data for 138 relatively bright carbon stars.

In total, we have compiled NIR photometric data for 694 relatively bright carbon stars. Total radiant energies were evaluated for these stars and distances of individual stars were determined. The derived SD distribution, projected onto the galactic plane, is shown in Figure 1. Since the observed NIR data were collected from several papers reported by different groups, the observations do not cover the Galaxy uniformly. However, the SD distribution obtained looks rather uniform within a circle with a radius of about $4 \mathrm{kpc}$ centered on the Sun.

\section{Space Distribution of Carbon Stars}

In order to investigate the space distribution of carbon stars in our entire Galaxy, more extensive NIR photometric observations including fainter stars are required. We carried out NIR photometry of carbon stars in the GCCS2 in October 1995. Since our observations were made from the northern hemisphere (from the Xinglong Station of the Beijing Astronomical Observatory United Laboratory of Optical Astronomy, Chinese Academy of Sciences), the area observed is limited to the longitude region between about $20^{\circ}$ and $160^{\circ}$. NIR data were obtained in the $J, H$, and $K$ bands for 470 stars. Distances for these 470 stars were estimated and the SD distribution has been derived. The total number of carbon stars for which distances have been estimated is 1164 including the relatively bright carbon stars. The SD distribution of these stars is shown in Figure 2. The paucity of stars in the range $60^{\circ} \leq l \leq 70^{\circ}$ and the higher stellar density in the longitude range $120^{\circ} \leq l \leq 160^{\circ}$ is due to an observational bias. Although 


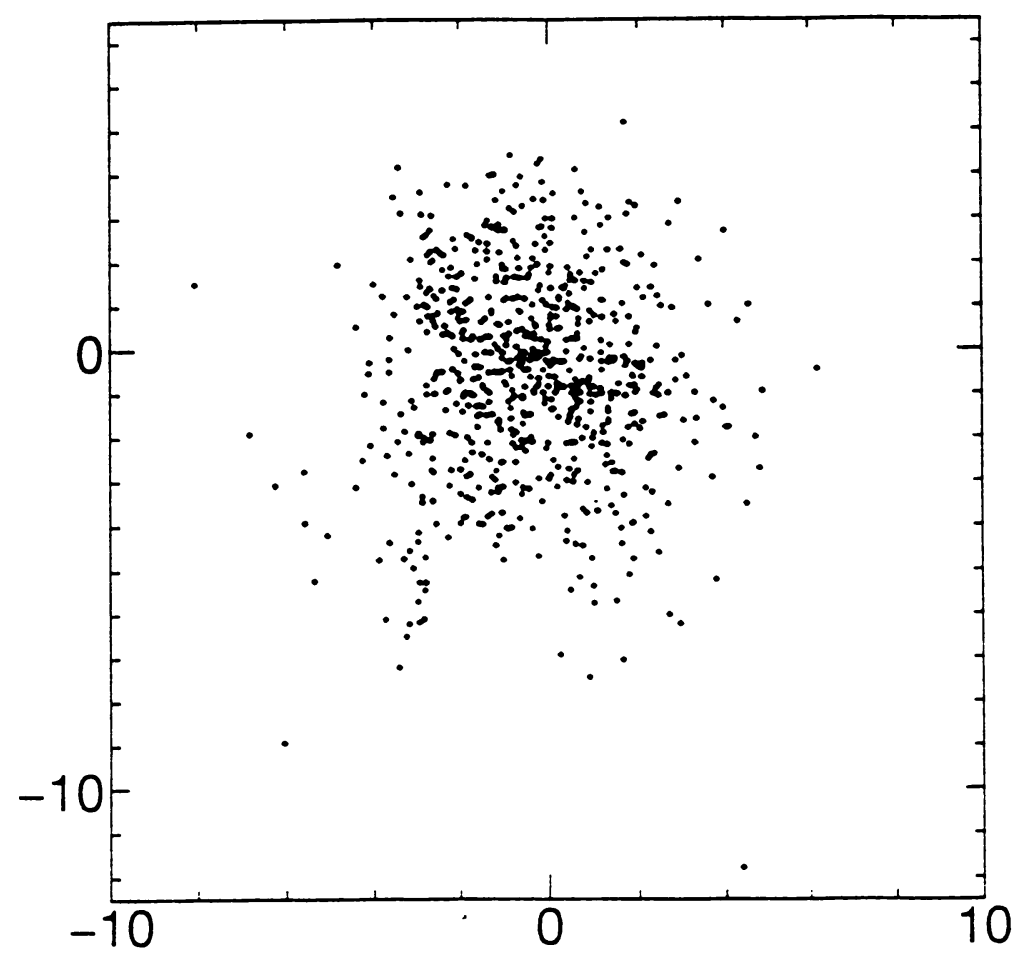

Figure 1. Space distribution of 694 relatively bright carbon stars projected onto the galactic plane. The Sun is located at the $(0,0)$ position and the Galactic Center is to the right. Distances derived by assuming luminosities of $10^{4} L_{\odot}$ are plotted in kpc.

the observed region does not cover sufficient area, the SD distribution of the carbon stars observed does not show a clear dependence on galactocentric distance. The reason for the pa: city of GCCS2 stars in the longitude range $-50^{\circ} \leq l \leq 50^{\circ}$ in the $l-b$ plane is still not clearly understood. More observations of carbon stars, especially in the galactic center direction, are required.

Aaronson et al. $(1989,1990)$ reported $J, H$, and $K$-band photometric data for carbon stars in the southern Milky Way (an additional 373 stars in the interval $238^{\circ} \leq l \leq 315^{\circ}$ ) and in the northern Milky Way (an additional 308 stars with $54^{\circ} \leq l \leq 185^{\circ}$ ). Although our present analysis does not include their data, they will be very valuable for future analysis.

The space distribution of $\mathrm{R}$ stars is different from that of $\mathrm{N}$ stars. However, the distribution examined in our present analysis is for the $\mathrm{R}$ and $\mathrm{N}$ stars combined. It will be important to examine the space distribution of $\mathrm{N}$ stars separately from $\mathrm{R}$ stars in future work. 


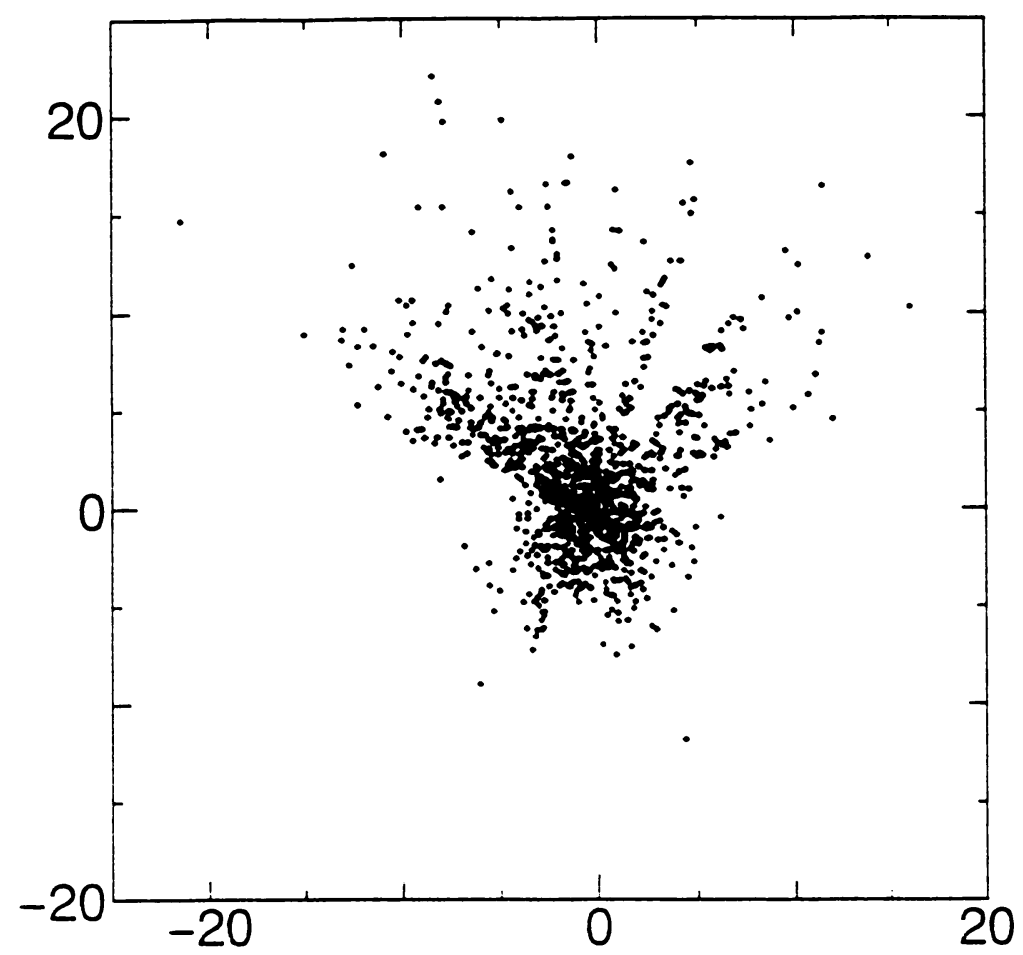

Figure 2. Space distribution of 1164 carbon stars in the GCCS2 catalog.

\section{References}

Aaronson, M., Blanco, V. M., Cook, K.H. \& Schechter, P. L. 1989, ApJ Supp., 70, 637

Aaronson, M., Blanco, V. M., Cook, K. H., Olszewski, E. W. \& Schechter, P. L. 1990, ApJ Supp., 73, 841

Catchpole, R. M., Robertson, B. S. C., Lloyd Evans, T.H. H., Feast, M. W., Glass, I. S. \& Carter, B. S. 1979, SAAO Circulars, 1, 61

Claussen, M. J., Kleinmann, S. G., Joyce, R. R. \& Jura, M. 1987, ApJ Supp., 65, 385

Epchtein, N., Le Bertre, T. \& Lépine, J. R. D. 1990, $A \& A, 227,82$

Epchtein, N., Le Bertre, T., Lépine, J.R. D., Marques dos Santos, P., Matsuura, O. T. \& Picazzio, E. 1987, A\&A Supp., 71, 39

Groenewegen, M.A.T., de Jong, T., van der Bliek, N.S., Slijkhuis, S. \& Willems, F. J. 1992, $A \& A, 253,150$

Guglielmo, F., Epchtein, N., Le Bertre, T., Fouqué, P., Hron, J., Kerschbaum, F. \& Lépine, J. R. D. 1993, A\&A Supp., 99, 31

Jura, .M. 1990, in From Miras to Planetary Nebulae: Which Path for Stellar Evolution?, ed. M. O. Mennessier and A. Omont (Editions Frontières), p. 41

Jura, M. \& Kleinmann, S. G. 1989, $A p J, 341,359$

Jura, M. \& Kleinmann, S. G. 1990, ApJ, 364, 663

Noguchi, K., Kawara, K., Kobayashi, Y., Okuda, H., Sato, S. \& Oishi, M. 1981, Publ. Astron. Soc. Japan, 33, 373

Noguchi, K., Sun, J. \& Wang, G. 1991, Publ. Astron. Soc. Japan, 43, 275

Stephenson, C. B. 1989, Publ. Warner \& Swasey Obs., 3, No. 2 


\section{Discussion}

Hron: I would like to draw your attention to the ongoing near-infrared sky survey (DENIS) and a planned survey (2MASS) which will give us all the carbon stars in the Galaxy - but it will take time.

Noguchi: Thanks for your comment. 